\title{
Innovazioni gestionali e nuove relazioni sistemiche per la competitività dell'impresa
}

\author{
SERGIO SiLVESTRELLI*
}

\begin{abstract}
Obiettivo del paper: Il paper è diretto a porre in evidenza alcune tappe significative dell'evoluzione del pensiero economico sulle caratteristiche e sulle fonti dell'innovazione nell'impresa.

Metodologia: Analisi e confronto dei risultati di qualificate ricerche, svolte sull'economia e sulla gestione dell'innovazione aziendale a livello internazionale.

Risultati: Viene posto in rilievo che l'innovazione aziendale importante non è soltanto di natura tecnologica (attinente ai processi, ai materiali e ai prodotti), ma che essa può essere anche organizzativa, gestionale o nelle relazioni sistemiche esterne.

Limiti della ricerca: Un limite evidente è che il numero delle ricerche considerate non è esaustivo, rispetto ai molti autori che hanno analizzato nelle proprie pubblicazioni le problematiche trattate in questo paper.

Implicazioni pratiche: Il lavoro ribadisce che il vantaggio competitivo dell'impresa può essere fondato su innovazioni, non soltanto tecnologiche, ma anche organizzativo-gestionali e nel modello di business.

Originalità del lavoro: Lo studio riesce ad illustrare sinteticamente come si è sviluppato il concetto di innovazione, ai fini del miglioramento della competitività dell'impresa.

Parole chiave: rapporto fra innovazione e competitività; forme dell'innovazione aziendale; fonti dell'innovazione; innovazione nel business model.

Purpose of the paper: The paper highlights some significant milestones in the evolution of economic thought on the characteristics and sources of innovation in the enterprise.

Methodology: Analysis and comparison of results of qualified studies, conducted on the economy and corporate innovation management at an international level.

Findings: Important business innovation proves to be, not only of a technological nature (relating to processes, materials and products), but also organizational, managerial or connected with the external relations of firms.

Research limitations: A clear limitation is that the number of studies considered is not comprehensive with respect to the many authors who have analyzed the issues addressed in this paper.

Practical implications: The work confirms that the competitive advantage of the firm can be founded, not only on technological, but also on organizational-managerial and business
\end{abstract} model innovations.

* Ordinario di Economia e Gestione delle Imprese - Università Politecnica delle Marche e-mail: s.silvestrelli@unipvm.it

sinergie, rivista di studi e ricerche

n. 94, Maggio-Agosto 2014, pp. 3-26

ISSN 0393-5108-DOI 10.7433/s94.2014.02 
Originality of the paper: The study illustrates how the concept of innovation has been developed, in order to improve the competitiveness of firms.

Key words: relationship between innovation and competitiveness; forms of firm innovation; sources of innovation; innovation in the business model.

\section{Crescente importanza del fenomeno dell'innovazione nelle ricerche svolte nell'ambito delle scienze sociali}

La crescente rilevanza delle ricerche sull'innovazione nell'ambito delle scienze sociali è dimostrata oggettivamente dalla rapida crescita del numero di riviste internazionali, di associazioni professionali e di unità di ricerca (centri, istituti, dipartimenti universitari, ecc.), che si occupano di innovazione ${ }^{1}$.

Soprattutto negli ultimi anni si riscontra una crescita del numero di "ricerche scientifiche" su vari aspetti dell'innovazione, affrontati da studiosi di diverse discipline accademiche (economia, management, sociologia, geografia, storia, ecc.).

Prima del 1960 le pubblicazioni sull'innovazione sono state poche e lontane fra loro $^{2}$.

Si osservi la figura 1, nella quale viene riportata la percentuale di articoli, con il termine "innovazione" sul titolo della pubblicazione, sul totale degli articoli pubblicati ogni anno nel campo degli studi di scienze sociali, in base ai dati dell'ISI Web of Science e della British Library ${ }^{3}$ : il grafico mostra che l'interesse degli studiosi aumenta in modo costante solo a partire dagli anni sessanta, per subire una forte accelerazione negli anni novanta e duemila. In particolare, il grafico mostra che (in base dai dati dell'ISI Web of Science) il numero (in percentuale) delle pubblicazioni sul fenomeno dell'innovazione è passata tra il 1960 e il 2008 da circa

1 Nel presente paper si intende porre in evidenza l'evoluzione della configurazione dell'innovazione aziendale secondo un'ottica economico-manageriale, piuttosto che svolgere una esauriente rassegna dell'ampia letteratura economica sul fenomeno dell'innovazione.

2 L'unica eccezione è l'opera delle scienziato austro-americano Joseph Schumpeter "The theory of economic development", pubblicata in Germania nel 1912. Combinando insieme intuizioni derivanti dall'economia, dalla sociologia e dalla storia, questo studioso sviluppa un approccio molto originale allo studio dei cambiamenti di lungo periodo dell'economia e della società, basato sul ruolo centrale giocato dall'innovazione e dai fattori che la influenzano.

Le idee di Schumpeter iniziarono ad essere apprezzate solo a partire dagli anni sessanta (cioè dieci anni dopo la sua morte), quando divenne evidente fra gli economisti la limitata capacità dei modelli economici dominanti (basati sulla matematica e su esercizi di equilibrio statico) di spiegare i cambiamenti tecnologici, economici e sociali di lungo periodo (Fagerberg e Verspagen, 2009, p. 220).

3 Com'è noto l'ISI Web of Science è il più importante database bibliografico/citazionale multidisciplinare, che consente di effettuare ricerche tematiche in più di 12.000 riviste internazionali ad elevato impact factor e in 148.000 atti di convegni da tutto il mondo nel campo delle scienze, delle scienze sociali, delle arti e delle scienze umanistiche. 
$0,02 \%$ a $0,7 \%$ con un incremento di circa 35 volte. La forte rilevanza assunta dagli studi sull'innovazione costituisce pertanto un fenomeno storicamente recente (Fagerberg et al., 2012, 1146).

Oltre alla rapida crescita del numero di studi svolti, si riscontra anche una crescente varietà nell'articolazione delle strutture di ricerca e nelle modalità di realizzazione dei lavori scientifici. La crescente diversificazione e specializzazione della conoscenza contribuisce a rendere sfumati i confini tradizionali fra discipline nell'organizzazione della scienza.

Fig. 1: Numero (in \%) di articoli con il termine "innovazione" nel titolo sul totale degli articoli pubblicati annualmente nell'ambito delle scienze sociali dal 1956 al 2008

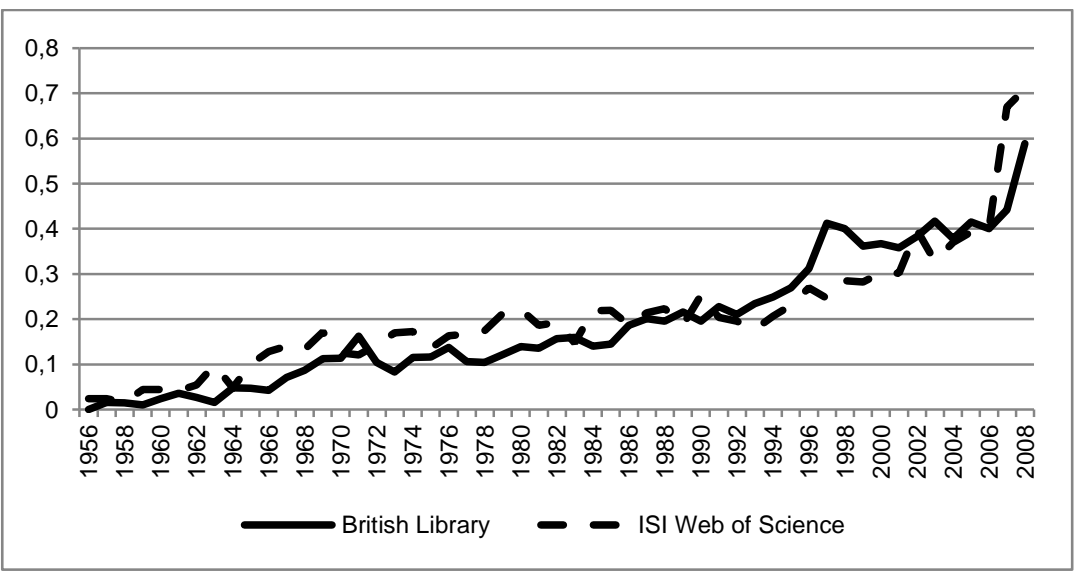

Fonte: Fagerberg et al., 2012, p. 1133 (in base ai dati ottenuti dall'ISI Web of Science e dalla British Library)

Oltre ad individuare in una prospettiva storica i principali contributi scientifici sull'innovazione, alcuni autori (Fagerberg et al., 2012) hanno analizzato l'utilizzazione di questi contributi da parte di studiosi di diverse discipline scientifiche, mediante l'esame delle citazioni (di tali contributi) presenti negli articoli scientifici. La figura n. 2 indica l'evoluzione della quota di citazioni realizzate nelle pubblicazioni dagli studiosi dei primi dieci gruppi di discipline scientifiche, individuate nello studio, sul totale delle citazioni relative ai principali lavori sull'innovazione (cioè alla cosiddetta "core literature") ${ }^{4}$.

4 I contribuiti scientifici fondamentali sull'innovazione, individuati da Fagerberg et al. (2012, p. 1144) e riportati in ordine decrescente delle citazioni ricevute in ogni periodo, sono in seguenti :

a) prima del 1969: 1) Roger E.M, Diffusion of Innovations, 1962; 2) Schumpeter J.A., The theory of Economic Development, 1934; 3) Arrow K., Economic welfare and the allocation of resource for invention, 1962; 4) Schumpeter J.A., Capitalism, Socialism and Democracy, 1942; 5) Burns T., Stalker G.M., The management of innovation, 1961; 
Fig. 2: Evoluzione del numero (in \% sulle citazioni totali) delle citazioni della "core literature" sull'innovazione nelle pubblicazioni dei primi dieci gruppi di "discipline scientifiche" dal 1950 al 2009

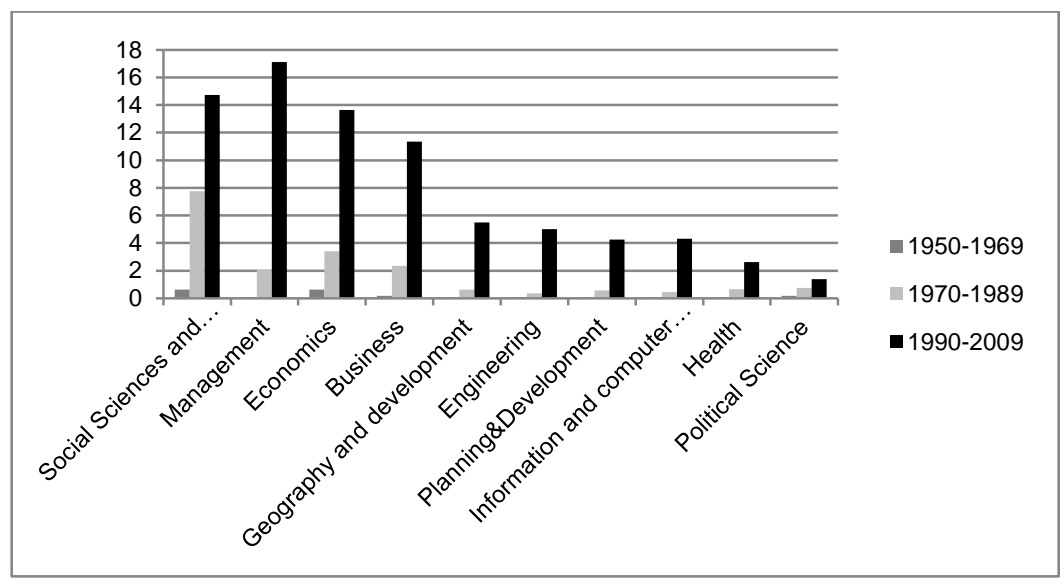

Fonte: Fagerberg et al., 2012, 1145.

Dai dati emerge che prima del 1970 le citazioni erano poche e lontane fra loro e tendevano ad essere concentrate nell'ambito dei gruppi disciplinari delle scienze sociali e umanistiche e dell'economia ${ }^{5}$. Nei decenni successivi esse subiscono una crescita costante e si estendono in molti gruppi di discipline scientifiche, che vanno oltre quelli strettamente sociali (si pensi all'ingegneria e all'information and computer science). Ciò che colpisce di più l'attenzione è tuttavia il rapido aumento del numero in \% delle citazioni realizzate nei lavori di management, che, da una quota molto bassa nel periodo precedente gli anni settanta, hanno assunto un ruolo centrale negli ultimi due decenni (Fagerberg et al., 2012, p. 1146).

Negli ultimi anni gli studiosi di management hanno mostrato un crescente interesse per le problematiche dell'innovazione, citando spesso nelle proprie

b) dal 1970 al 1989: 1) Nelson R, Winter S., An evolutionary theory of economic change, 1982; 2) Freeman C., The economics of Industrial Innovation, 1984; 3) Pavitt K., Sectorial patterns of technical change, 1984; 4) Freeman C., Technology policy and economic performance, 1987; 5) Von Hippler E., The source of innovation, 1988;

c) dal 1990 al 2008: 1) Neson R., National innovation system: a comparative study, 1993;

2) Porter M., The competitive advantage of nations, 1990; 3) Lundvall B.A., National system of innovation, 1992; 4) Cohen W., Levinthal D., Absorptive capacity: a new perspective on learning and innovation, 1990; 5) Saxenian A., Regional advantage, 1994.

5 Nell'ambito del gruppo delle "scienze sociali e umanistiche" Fagerberg et al. (2012) raggruppano diversi ambiti scientifici di natura affine, quali la sociologia, la psicologia, il diritto, le relazioni internazionali, l'antropologia, la storia, la filosofia, ecc., mentre nel gruppo delle discipline di "business" sono comprese le discipline di economia aziendale e la finanza aziendale. 
ricerche importanti lavori di natura economica ${ }^{6}$. Viceversa vari economisti hanno richiamato nei propri lavori studi di management. Emerge pertanto una forte convergenza (e un terreno comune) fra gli studi sull'innovazione di matrice economica e quelli di management. Ciò ha dato vita ad una "contaminazione culturale", che ha avuto effetti positivi, sia sugli studi degli economisti politici, sia su quelli degli economisti d'impresa ${ }^{7}$.

\section{Cenni sull'innovazione tecnologica: aspetti economico-gestionali}

Gli economisti che hanno studiato l'innovazione hanno approfondito in passato i fattori, le caratteristiche e gli effetti delle innovazioni nelle tecnologie attinenti ai processi produttivi, ai materiali e ai prodotti ${ }^{8}$.

Gli studiosi di management hanno da tempo rilevato che la pianificazione e la realizzazione dell'innovazione tecnologica nell'impresa costituiscono un complesso processo decisionale, nell'ambito del quale assumono un particolare rilievo le "interrelazioni economiche ed organizzative" tra le principali funzioni gestionali, quali la R e S, la Produzione e il Marketing ${ }^{9}$.

6 È stato a tale proposito rilevato che gran parte dei più importanti contributi sull'innovazione tendono a non essere richiamati nelle pubblicazioni su riviste di natura economica. Un esempio interessante è il libro di Nelson e Winter del 1982 (An Evolutionary theory of economic change), che rappresenta uno dei contributi scientifici più citati.

Particolarmente sorprendente è l'aspetto seguente: il lavoro di Nelson e Winter ha ricevuto più citazioni in articoli pubblicati in riviste di studi organizzativi e di management, che in articoli su riviste di economia, la quale rappresenta il background culturale degli autori (Fagerberg e Verspagen, 2009, p. 229).

7 Il principale incentivo a sviluppare gli studi sull'innovazione - particolarmente in Europa appare riconducibile al riconoscimento del rilevante impatto, che l'innovazione può avere sull'economia e sulla società, e alla necessità di accrescere le conoscenze sul ruolo che la politica economica e industriale dell'Operatore Pubblico può avere nel favorire l'innovazione. Secondo Fagerberg e Verspagen (2009, p. 229), questa focalizzazione sull'aspetto di policy ha forse contribuito a non attrarre nelle discipline manageriali lo stesso interesse per l'innovazione, riscontrato negli studi di matrice economica.

8 Quando la rivista "Sinergie" all'inizio degli anni novanta sviluppò nuovi indirizzi di ricerca, ampliò il comitato scientifico e la redazione, alcuni interessanti contributi, attinenti alla gestione strategica dell'innovazione tecnologica nell'impresa, vennero presentati nel n. 23 del 1990 e nei n. 24 e 25/26 del 1991 della nuova versione della rivista, la cui pubblicazione è stata curata da Sergio Silvestrelli.

9 Riconoscendo che "prodotto" e "processo" non sono percorsi innovativi contrapposti, ma elementi di un fenomeno unitario, si giunge a sostenere sostanzialmente che nei processi innovativi sono comunque coinvolte tutte le aree aziendali. Oltre a svolgere un ruolo nel "processo innovativo globale", le aree funzionali d'impresa forniscono contributi ad ogni stadio del processo stesso, data l'interdipendenza tra $i$ vari 'steps' dell'innovazione aziendale.

Nonostante possa sussistere il primato relativo di una funzione è sempre e comunque utile 
L'innovazione tecnologica, intesa come processo economico-organizzativo, presenta un elevato grado di complessità.

Tale complessità può essere colta tramite i concetti seguenti:

a) la "multidimensionalità", la quale indica che l'innovazione non riguarda soltanto la tecnologia della produzione manifatturiera, ma anche le altre attività aziendali, tenendo presente che l'impresa, considerata come "insieme di attività", si presenta come un "insieme di tecnologie" (Porter, 1987);

b) la "globalità" dell'innovazione, nel senso che il processo innovativo va gestito come un fenomeno unitario nell'ambito dell'impresa tramite un approccio organizzativo $^{10}$

La complessità dei processi aziendali di innovazione risulta evidente nel momento in cui vengono superate alcune tradizionali e semplicistiche distinzioni, adottate dagli studiosi da vari decenni.

Si pensi, ad esempio, alle classificazioni delle innovazioni in innovazioni di "prodotto" o di "processo", "radicali" o "incrementali", "spinte dalla tecnologia" o "guidate dalla domanda": esse presentano innanzitutto il limite (oggettivo) di identificare i soli estremi di situazioni, che in realtà si pongono lungo "un continuum", difficilmente frazionabile; esse mostrano inoltre una capacità interpretativa decrescente, di fronte alle attuali evoluzioni tecnologiche ed a nuovi metodi di gestione dell'innovazione tecnologica.

Un'osservazione fondamentale riguardo al ruolo dell'innovazione tecnologica nella competizione aziendale è questa: considerando che una caratteristica essenziale dei sistemi di impresa è costituita dalla creazione di valore aggiunto, occorre riconoscere che qualsiasi attività aziendale (creatrice di valore) richiede l'utilizzazione di tecnologia.

Il concetto di tecnologia può quindi essere interpretato in maniera ampia, riconoscendo che la «tecnologia» è incorporata in ogni attività del sistema d'impresa (Golinelli, 2000), ed è altresì incorporata nelle stesse risorse utilizzate per lo svolgimento delle attività aziendali.

c) Ma la tecnologia non riguarda soltanto le singole imprese, poiché essa caratterizza l'intero sistema inter-aziendale del valore: oltre ad essere estremamente pervasiva all'interno essa è collegata alla tecnologia dei fornitori,

far riferimento simultaneamente alle conoscenze e competenze delle altre aree gestionali.

10 Qualificati studi degli anni ottanta sul processo, attraverso cui le imprese generano, sviluppano e commercializzano le innovazioni tecnologiche, hanno posto in evidenza una aspetto rilevante: il processo innovativo dipende da interazioni e feed-back sistematici tra i diversi stadi della ricerca, nonché tra la ricerca, la produzione e il marketing.

Stephen Kline e Nathan Rosenberg (1986) contrappongono al "modello lineare" il cosiddetto chain-linked model (o "modello concatenato"). In questo modello, la capacità dell'impresa di generare, sviluppare e commercializzare le innovazioni dipende da un processo di interazione complesso che coinvolge direttamente ricerca, produzione e marketing. Tra queste funzioni non vi è relazione «a cascata» dalla ricerca, alla produzione, al marketing. Persino all'interno della stessa funzione di R\&S, non si assiste ad una catena lineare diretta, che conduce dai risultati della ricerca di base, alla ricerca applicata in laboratorio, allo sviluppo di un nuovo prodotto (Gambardella, 1999). 
dei distributori e degli stessi acquirenti (Porter, 1987, p. 61). Si consideri, ad esempio, i sistemi dell'I.C.T.: la tecnologia è il substrato di tutta la catena del valore, poiché ogni attività creatrice di valore utilizza e genera informazioni, ed inoltre risulta di fondamentale importanza nello stabilire i collegamenti tra attività di ogni tipo, sia all'interno dell'impresa sia tra imprese diverse.

In definitiva, come è stato dimostrato da studiosi e da manager, il progresso tecnologico non è rilevante in quanto tale, ma lo è nella misura in cui riesce ad innalzare la capacità competitiva dell'impresa e (di conseguenza) a sviluppare il sistema economico-industriale.

\section{Dall'innovazione tecnologica all'innovazione organizzativa nell'impresa}

La complessità del fenomeno innovativo si estrinseca nelle diverse "tipologie" di innovazione, che possono essere realizzate da un'impresa. Infatti, oltre alle innovazioni nei prodotti, nei processi produttivi e nei materiali, che interessano prevalentemente le aree della $\mathrm{R} \& \mathrm{~S}$, della produzione e degli approvvigionamenti, il miglioramento del livello della "competitività aziendale" può fondarsi anche su innovazioni "soft", che riguardano elementi "immateriali", come le strutture organizzative, le attività di marketing, la gestione finanziaria, fino ad arrivare all'intero "modello di business" di un'impresa ${ }^{11}$. Si tratta di innovazioni definite dalla letteratura "non tecniche" (Camison e Villar-Lopez, 2011, p. 1300).

Non è raro osservare nella realtà che queste forme di innovazione si realizzano contemporaneamente nella stessa impresa, poiché sono interdipendenti tra di loro. Ad esempio, studiando il caso di un'impresa familiare operante nel settore della metallurgia, alcuni autori (Calia et al., 2007, p. 432), mostrano come l'impatto di un'innovazione tecnologica non si limiti all'introduzione di nuove funzionalità nel prodotto, ma possa estendersi a significativi cambiamenti nelle attività operative e commerciali dell'impresa innovatrice, le quali vanno a configurare un nuovo modello gestionale in aziende di qualsiasi dimensione ${ }^{12}$.

11 Alcuni aspetti significativi delle implicazioni di mercato e delle nuove logiche concorrenziali, derivanti dalle innovazioni aziendali, sono stati presentati in un precedente lavoro (Silvestrelli, 2004).

12 Già negli anni novanta uno studio interessante ha mostrato che, nel caso delle PMI, ad influire maggiormente sulla performance non è l'innovazione di prodotto o di processo, ma il più generale "orientamento all'innovazione" dell'impresa, inteso come positiva attitudine verso il cambiamento, come atteggiamento culturale ed organizzativo favorevole allo sviluppo di nuove idee, alla sperimentazione, alla creatività (Lumpkin e Dess, 1996, p. 142).

Inoltre, una meta-analisi recente (Rosenbush et al., 2011) sul rapporto fra innovazione e performance nelle piccole e medie imprese, ha sottolineato che l'orientamento all'innovazione dell'organizzazione ha effetti significativamente maggiori sulle prestazioni economiche delle imprese, rispetto allo sviluppo di prodotti innovativi. 
Più in generale, viene sostenuto che nelle realtà aziendali esiste una "complementarità" nelle "forme" dell'innovazione e negli "effetti" dell'innovazione.

Tale conclusione viene avvalorata anche dalle ricerche di Camison e VillarLopez (2014, p. 2898) sulle imprese spagnole, i quali hanno verificato che "l'innovazione organizzativa" favorisce in modo diretto lo sviluppo di "innovazioni di processo" e, in modo indiretto, la realizzazione di innovazioni di prodotto (ovvero mediato dall'innovazione di processo). L'introduzione di nuove pratiche manageriali, di nuovi assetti organizzativi, di nuove procedure, oltre che influire in modo positivo sulla performance aziendale, crea anche le condizioni favorevoli per realizzare l'innovazione tecnologica: tra innovazioni organizzative e innovazioni tecnologiche possono crearsi importanti sinergie.

Com'è noto, l'innovazione organizzativa consiste nell'introduzione di nuovi metodi organizzativi, sia nella gestione delle attività aziendali (nelle strutture operative), sia nella relazione fra un'impresa e altri attori esterni (OECD, 2005, citato da Camison, Villar-Lopez, 2014).

È opinione largamente condivisa che l'innovazione organizzativa rappresenti oggi una delle più importanti e sostenibili fonti di vantaggio competitivo per le imprese per la sua natura context-specific (Hamel, 2006, 2007, 2009), anche se la comprensione di questa forma di innovazione è ancora limitata, secondo una recente ricerca $^{13}$

\section{L'innovazione del "business model": dalla value innovation all'innovazione strategica}

È soprattutto a partire dalla metà degli anni novanta che si inizia a parlare di “Business Model”, sotto la spinta dell'avvento di Internet, il quale:

- da un lato, ha modificato in maniera radicale le modalità di competere delle imprese "tradizionali" di intere industrie (come quella musicale ed editoriale);

13 Camison e Villar-Lopez (2014) sostengono che pochi contributi concettuali e metodologici hanno affrontato il tema dell'analisi dell'innovazione organizzativa (Armbruster et al., 2008). Negli ultimi anni è aumentato il numero di studi sullo sviluppo delle innovazioni organizzative e sui fattori che favoriscono questo sviluppo. Sui fattori antecedenti dell'innovazione manageriale si veda: Damanpour e Aravind, 2011. In tale articolo viene realizzata un'analisi delle definizioni di innovazione organizzativa utilizzate in studi precedenti (dal 1978 al 2011) e viene scelta quella proposta dall'OECD (2005). Alcuni studi recenti hanno enfatizzato la natura complementare fra le innovazioni organizzative e tecnologiche (Battisti e Stoneman, 2010; Damanpour et al., 2009; Martinez-Ros e Labeaga, 2009; Piva et al., 2005), mostrando che le sinergie fra i due tipi di innovazione le rendono complementari piuttosto che sostitutive. La survey di Camison e Villar-Lopez (2014) su un campione di 144 imprese industriali spagnole conferma l'ipotesi che le innovazioni organizzative favoriscono lo sviluppo di capacità di innovazione tecnologica dei prodotti e dei processi e che entrambi questi elementi possono portare ad una performance superiore dell'impresa. 
- dall'altro lato, ha creato nuovi assetti organizzativi e nuove strategie competitive, riferite alle imprese operanti esclusivamente online.

Come è stato osservato (Teece, 2009, p. 175), il concetto di "business model" non ha un fondamento teorico ben definito, né negli studi di economia, né in quelli di management; esistono soltanto alcuni limitati riferimenti negli studi organizzativi: le forme organizzative sono una componente del "modello di business", ma non lo esauriscono.

All'inizio degli anni duemila gli autori Amit e Zott (2001, p. 511) hanno affermato che il "business model" è "il contenuto, la struttura e il governo delle transazioni tra un'impresa e i suoi partner esterni".

Questo comprende infatti tutte le modalità, mediante le quali un'impresa crea e trasferisce valore ai clienti, ottenendo un profitto da questa sua attività: esso pertanto si riferisce all'architettura organizzativa e finanziaria di un'azienda (Teece, 2009 , p. 173). In altre parole, esso descrive: i benefici che l'impresa crea per i clienti; come essa è organizzata per trasferirli; e come essa cattura parte del valore che trasferisce al mercato.

Un concetto simile a quello precedente, è la "value innovation", che è stato introdotto da Kim e Mauborgne (1997, 1999), osservando le caratteristiche delle imprese di successo. Gli studiosi hanno verificato che l'unico elemento comune a tutte le imprese osservate era relativo al modo di concepire la strategia: il punto di partenza non è la concorrenza (e quindi le modalità per superare la performance dei concorrenti), ma gli acquirenti e cioè la creazione di un valore superiore per $i$ clienti, tale da rendere "irrilevante" la competizione e formare uno spazio di mercato "incontestato" ("strategia oceano blu"); alcuni elementi salienti sono presentati nella tabella 1 .

Ma una delle definizioni più esaustive e dettagliate è stata proposta da Cheesbrough e Rosembloom (2002, p. 533), specificando che un "business model" ̀̀ caratterizzato dalla seguenti "scelte strategiche", assunte dal management aziendale:

1. articolare la "value proposition" per i clienti;

2. identificare un "segmento di mercato" e specificare i meccanismi tramite i quali ottenere i ricavi. Con l'innovazione, si tratta di ridefinire la base di clienti ai quali rivolgere la propria offerta, individuando un segmento di mercato "nascosto", ma sufficientemente ampio da essere profittevole: questo è stato il caso della Canon, che individuò un nuovo gruppo di clienti nei singoli consumatori e nelle piccole imprese, in un'epoca in cui le fotocopiatrici erano impiegate quasi esclusivamente dalle grandi organizzazioni;

3. definire la struttura della "catena del valore" necessaria, per offrire quella value proposition e gli asset complementari;

4. stimare la struttura dei costi e il profitto potenziale ritraibile dall'offerta. Da questo punto di vista il modello di business è necessario, per trarre profitto da un'innovazione tecnologica; ovviamente, ciò è più frequente nel caso di innovazioni "radicali" o comunque abbastanza significative. Viene comunque osservato che "una tecnologia mediocre, con un business model efficace, può 
essere più profittevole di una tecnologia eccellente, con un business model mediocre" (Chesbrough, 2010, p. 354);

5. descrivere la posizione dell'impresa nel "network di valore", che lega l'azienda ai fornitori e ai clienti, includendo anche l'identificazione dei potenziali competitors;

6. formulare la strategia competitiva.

Tab. 1: Elementi economici salienti della "value innovation"

Il focus dell'innovazione di valore non è la tecnologia, ma piuttosto la riconfigurazione del modello di business, al fine di creare un nuovo e superiore valore per il cliente; il suo obiettivo non è tanto quello di soddisfare in modo più efficace o più efficiente gli attuali clienti, quanto soddisfare una "domanda nuova", offrendo un prodotto, che sia contemporaneamente più economico e di maggior valore rispetto a quelli esistenti. Elemento tipico dell'innovazione di valore è proprio quello di superare il classico trade off tra differenziazione ed efficienza.

Realizzare un'innovazione di valore non è semplice, in quanto molteplici possono essere le barriere al cambiamento, non soltanto all'interno dell'impresa innovatrice, ma anche in riferimento alle altre imprese che fanno parte della sua supply chain. Si stratta soprattutto di barriere di natura culturale, attinenti agli schemi mentali, alle relazioni e ai processi consolidati che sono spesso difficili da scardinare (Matthyssens et al., 2006, p. 758).

Un esempio di value innovation si è realizzato nel corso degli anni duemila nel settore orafo, con la nascita del mercato della fashion jewellery, ossia del "gioiello moda", ad opera di imprese come Morellato e Bros Manifatture. Tale innovazione si fonda sulla ridefinizione del "concetto di prodotto": da "oggetto prezioso" - il cui valore dipende prima di tutto dalla qualità delle materie prime utilizzate - a "oggetto style symbol" - il cui valore dipende in misura significativa dal contenuto emozionale e simbolico, che si esprime tramite il design del bene. Tale nuovo gioiello ha dato vita ad un" nuovo mercato", formato, sia dai consumatori, che per ragioni di reddito non potevano prima permettersi l'acquisto di un gioiello classico, sia dai consumatori che erano alla ricerca di un bene diverso e più moderno.

L'innovazione di valore consiste quindi nell'aver creato un maggior valore per questi consumatori, superando il trade off tra costi e differenziazione: l'impiego di materiali meno costosi e l'efficienza nei processi produttivi, hanno consentito infatti di offrire il prodotto ad un prezzo nettamente inferiore, rispetto a quello dei gioielli tradizionali.

Un aspetto su cui convergono gli studiosi è che una modifica nel modello di business può costituire di fatto un'innovazione originale e profittevole ${ }^{14}$.

Un nuovo "modello di business" può costituire da solo una fonte di profitto, qualora sia sufficientemente differenziato (e quindi difficile da imitare), ed efficace rispetto alle caratteristiche dei clienti e dell'ambiente competitivo (Shlegelmich et al., 2003). Ciò si è verificato, ad esempio, nel caso della Dell, la quale non ha apportato nessun miglioramento profondo di natura tecnologica al prodotto - il personal computer - ma ha innovato il modo di vendere ai consumatori, dando loro

14 Sawhney et al. (2006) hanno adottato il concetto di "innovazione di business" (business innovation) per indicare la creazione di nuovo valore per $i$ clienti e per l'impresa, mediante il cambiamento di una o più dimensioni del sistema di business. Essi propongono uno strumento, definito "radar dell'innovazione", per delineare tutte le dimensioni, che le imprese possono considerare, per individuare nuove opportunità di innovazione e per confrontare la propria posizione (sulle dimensioni individuate), rispetto a quella delle imprese concorrenti. 
la possibilità di configurarsi da soli il prodotto da acquistare e vendendo direttamente senza intermediari.

Nel caso particolare della Dell il concetto di nuovo modello di business si sovrappone a quello di "innovazione strategica", la quale si è estrinsecata in una scelta fondamentale del modello di business (Govindarajan e Gupta, 2001, p. 4): la ridefinizione della catena del valore, cioè la trasformazione delle attività che la compongono e delle interfacce tra di esse. Per attuare il passaggio da un canale distributivo indiretto ad uno diretto nella vendita di personal computer personalizzati ai consumatori, la Dell ha trasformato la propria catena del valore, esternalizzando la fabbricazione di tutte le componenti e mantenendo internamente soltanto l'assemblaggio del prodotto finito; ciò ha richiesto altresì lo sviluppo di relazioni solide sia con i fornitori, sia con i consumatori: è stata così realizzata un'integrazione economica "virtuale", senza "integrazione verticale delle attività" al proprio interno.

\section{Nuove relazioni sistemiche nel business model: strategia di "outsourcing" delle imprese e "disintegrazione verticale" del settore}

Una scelta strategica nella modifica del business model (fondato sulla specializzazione delle imprese) è stata rilevata da due autori (Christensen e Raynor, 2004) nell'analisi della ristrutturazione del settore informatico, avvenuta tra il 1978 e il 1990, ed estrinsecatasi in una disintegrazione verticale dell'industria.

"La figura 3 sintetizza in maniera semplificata le transizioni avvenute nel settore dei personal computer, mostrando come i sistemi integrati e le aziende verticalmente integrate che dominavano nei primi anni, quando la funzionalità dei prodotti era ancora insufficiente, abbiano lasciato il posto negli anni successivi a una popolazione non integrata e orizzontalmente stratificata di aziende. Si direbbe quasi che il settore sia passato in un tritacarne. L'andamento grafico sarebbe analogo per ognuno dei network di valore che compongono il settore. In tutti i casi, il driver della modularizzazione e della disintegrazione non è stato il passare del tempo, o la "maturazione" del settore di per sé. Ciò che guida questo processo è una sequenza casuale prevedibile" (Christensen e Raynor, 2004, p. 138).

"Mentre prima l'integrazione era una necessità competitiva, poi diventa uno svantaggio competitivo.

La figura 3 è semplificata, perché il modello di business integrato non è scomparso da un giorno all'altro; ha perso progressivamente il suo predominio man mano che la traiettoria di miglioramento della performance toccava i diversi segmenti di mercato, portando progressivamente al successo il modello modulare. Noi sottolineiamo il fatto che sono state le circostanze, ossia i gap e i surplus di performance, a determinare l'efficacia di queste strategie di architettura e di integrazione. Ciò significa ovviamente che se cambiano le circostanze, deve cambiare anche l'approccio strategico. Anzi, dopo il 1990, c'è stata una certa reintegrazione nel settore informatico"(Christensen e Raynor, 2004, p. 139). 
Fig. 3: La transizione dall'integrazione verticale alla stratificazione orizzontale del settore dei computer a microprocessori

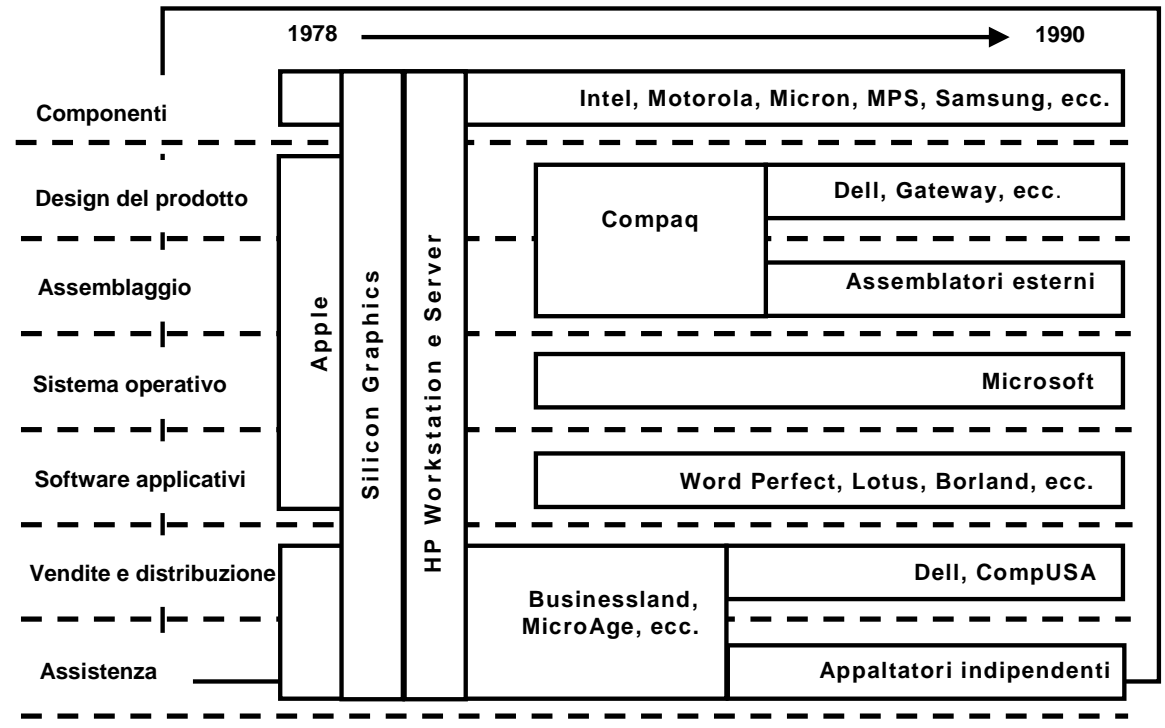

Fonte: Christensen e Raynor, 2004, p. 139.

"Oggi, però, le cose stanno cambiando ancora. Poiché l'informatica si incentra sempre di più su Internet, i sistemi operativi basati su architetture modulari (come Linux) e i linguaggi di programmazione modulari (come Java) costituiscono delle innovazioni scardinanti ibride rispetto a quelle di Microsoft. Questa modularità sta consentendo a tutta una serie di aziende specializzate di fare le prime incursioni nel settore" (Christensen e Raynor, 2004, p. 141).

Non può sfuggire che "il modello" delineato dai due autori coincide con uno "schema interpretativo" dello sviluppo delle imprese industriali (fondato sull'outsourcing) e della relativa ristrutturazione di molti settori industriali, che è stato formulato da economisti italiani già negli anni settanta (Silvestrelli, 1978).

Le caratteristiche principali di tale modello di sviluppo sono sinteticamente indicate nella tabella 2.

Questo approccio innovativo al modello di business da vari decenni si è diffuso (tramite la strategia di decentramento produttivo) in numerose aziende di qualsiasi dimensione e in molti settori industriali (Varaldo, 1979); il fenomeno si riscontra ormai in quasi tutti i paesi, sia avanzati, sia in via di sviluppo (Gregori, 2001).

"Global sourcing" e "outsourcing" sono di fatto diventati due fenomeni strutturali nelle economie moderne. 
Tab. 2: Modello di sviluppo industriale "per disintegrazione verticale del settore"

Sulla base delle proprie risorse e competenze distintive, le imprese più innovative hanno attuato una riallocazione delle risorse finanziarie ed organizzative, diretta a sviluppare e a internalizzare le funzioni operative strategiche e più profittevoli, come la progettazione, l'assemblaggio e la commercializzazione dei prodotti. Esse hanno altresì realizzato una politica produttiva fondata sulla segmentazione dei processi, sul decentramento delle fasi di lavoro standardizzate e sulla subfornitura di componenti speciali.

Inteso come un nuovo «modo di produrre», l'outsourcing ha esplicato i suoi effetti economici, non solo sull'organizzazione interna delle singole imprese, ma anche sulla organizzazione del settore produttivo nel suo complesso. Esso è diventato un fattore strutturale dello sviluppo economico, dando origine ad un processo di "disintegrazione verticale", che si è concretato nella nascita di nuovi sub-settori produttivi di componenti modulari nell'ambito della medesima industria.

Tale fenomeno ha inoltre reso possibile il conseguimento delle economie di scala tecnologiche ai diversi livelli verticali del processo produttivo globale. Di conseguenza, molti stabilimenti industriali sono stati costituiti con la capacità produttiva più efficiente, senza assumere necessariamente una grande dimensione, proprio perché il processo di lavorazione (da essi svolto) comprende un relativamente esiguo numero di fasi operative. Configurandosi come divisione del lavoro tra imprese "in senso smithiano", l'outsourcing di attività ha favorito la specializza-zione produttiva in senso verticale delle aziende, ha facilitato la diffusione del progresso tecnico ed ha promosso consistenti aumenti della produttività del lavoro.

Fonte: Silvestrelli, 1978.

Dall'osservazione della realtà emerge che sempre più frequentemente queste due scelte strategiche si combinano: l'impresa decide di esternalizzare alcune delle attività, precedentemente svolte al proprio interno, a soggetti che si trovano in Paesi esteri (offshore outsourcing). Ad essere interessate da tale fenomeno non sono soltanto le attività di produzione, ma anche attività infrastrutturali e la R\&S; molte aziende, ad esempio, hanno spostato all'estero i servizi legati all'ICT, con un focus particolare sull'India (Nanut e Tracogna, 2003, p. 27). Va infatti rilevato che da decisioni di natura operativa - volte a ridurre i costi di fabbricazione dei prodotti tali decisioni assumono oggi una rilevanza strategica, andando ad interessare anche attività vicine alle "core competencies" aziendali, per ottenere benefici ulteriori, rispetto al mero risparmio nei costi. Tali benefici sono (Contractor et al., 2010, p. $1427)^{15}$ :

- acquisizione di conoscenze e di esperienze non possedute internamente, accesso a competenze e a talenti altrimenti non disponibili;

- migliore comprensione dei mercati esteri, che può servire anche ad una migliore penetrazione commerciale.

A livello macro le conseguenze della strategia di offshore outsourcing possono determinare la riorganizzazione su scala internazionale di intere industrie (come nel caso dell'elettronica, delle telecomunicazioni, dell'automobile) (Di Gregorio et al., 2009). In questo nuovo scenario sempre più critico sarà il ruolo della logistica, quale attività indispensabile per collegare le attività a livello globale (Dagnino et al., 2011).

15 Si tratta delle attività definite "essenziali", cioè attività ad alto valore, complementari rispetto alle attività "core" (ad esempio, la sperimentazione nello sviluppo di un nuovo farmaco per le imprese farmaceutiche). 
A livello micro (di singola impresa) la scelta strategica di esternalizzare all'estero le attività determina una riconfigurazione della "catena del valore aziendale" in forma disaggregata e dispersa geograficamente, fino ad arrivare a casi estremi nei quali all'interno dell'impresa viene mantenuta soltanto la gestione commerciale del brand ed esternalizzato tutto il resto. In tali casi è evidente che l'intero "modello di business" si trasforma e che il management si trova a ripensare anche le strutture organizzative e i processi (Lampel e Bhalla, 2011, p. 348).

Nell'economia globalizzata di oggi le imprese, piuttosto che ragionare su singole e specifiche attività della catena del valore (approccio atomistico), tendono a ragionare in modo sistemico sull'assetto dell'intera catena del valore (approccio olistico) (Schmeisser, 2013).

È bene tuttavia tener presente che i benefici, ottenibili con tali strategie, possono ad un certo punto essere bilanciati (se non addirittura superati) dai costi, derivanti dall'incremento della complessità nella gestione e nel coordinamento di tutte le relazioni interaziendali. Peraltro, tale aspetto risulta particolarmente importante nella gestione delle PMI distrettuali (Varaldo, 2006).

\section{La pluralità delle fonti dell'innovazione tecnologica}

La letteratura economica sulle fonti dell'innovazione è molto ampia e non può essere esaminata in questa sede. Mi limito a ricordare sinteticamente che la teoria economica ha offerto inizialmente due diversi modelli esplicativi sull'origine dell'innovazione tecnologica:

- l'approccio demand pull, secondo il quale la causa principale di un cambiamento tecnologico è data dal riconoscimento da parte delle imprese di bisogni insoddisfatti del mercato;

- l'approccio technology push, per il quale l'ambiente scientifico-tecnologico genera nuove tecnologie in modo autonomo e indipendente dall'andamento del mercato.

Ricerche successive hanno attenuato la dicotomia tra modelli demand pull e quelli technology push, considerando le relazioni di interdipendenza che legano mondo scientifico e mondo economico. Una recente analisi ha esaminato i cento articoli più rilevanti, che sono stati pubblicati sulle fonti dell'innovazione nelle riviste internazionali dal 1956 al 2010 (Di Stefano et al., 2012). Tale contributo, oltre a confermare l'importanza della tecnologia come fonte dell'innovazione e chiarire il ruolo sempre più rilevante della domanda, ha attribuito una valenza particolare alle risorse, alle competenze e alle conoscenze sviluppate nell'impresa nel favorire una sintesi fra i due orientamenti ${ }^{16}$.

16 Molte innovazioni tecnologiche hanno origine nell'ambiente scientifico e hanno comunque bisogno di un mercato e delle relative risorse e competenze complementari, per poter essere commercializzate con successo. Analogamente, le innovazioni che provengono da una prospettiva di tipo esclusivamente "demand pull" necessitano di adeguate competenze tecniche per poter essere effettivamente realizzate. La maggior parte 
Un ulteriore passo verso la comprensione delle fonti dell'innovazione, nell'ambito di una data traiettoria tecnologica, è stato fatto con il contributo di Von Hippel (1978; 1987), il quale si è incentrato sull'individuazione dei soggetti/imprese che sviluppano l'innovazione. Egli classifica le imprese in base al rapporto, che quelle hanno con l'innovazione, grazie al quale si traggono vantaggi economici: utilizzo, produzione, fornitura (Von Hippel, 1990, p. 231).

La tesi di Von Hippler è che il processo innovativo è un'attività svolta da diversi soggetti: non solo il produttore (ad esempio la grande impresa), ma anche l'utilizzatore e il fornitore. Viene così sottolineata l'importanza, non solo delle fonti interne di innovazione, ma anche di quelle esterne ai confini organizzativi aziendali.

Non può sfuggire che tali ricerche rispecchiano l'evoluzione del sistema industriale verso forme di collaborazione tra imprese. All'aumentare del grado di complessità del processo innovativo, aumenta anche l'esigenza per la singola impresa di gestire le relazioni con altri soggetti, le cui "competenze specifiche" sono funzionali all'innovazione. Pertanto, la capacità di apprendere dall'esperienza - non solo interna ma anche di fornitori, clienti e concorrenti - è una strategica fonte di innovazione (Pavitt, 1991) ${ }^{17}$.

\section{Un nuovo metodo di ricerca e gestione dell'innovazione: l'open innovation}

In una prospettiva manageriale di generazione e di applicazione di nuove idee, l'open innovation è stata proposta come un nuovo "paradigma” per la gestione

degli articoli considerati mostrano che le risorse, le competenze e le conoscenze sono alla base di questa sintesi fra le due fonti di innovazioni. Spesso inoltre esse stesse possono rappresentare una fonte di innovazione quando sono in grado di consentire all'impresa di assorbire nuovi segnali tecnologici e/o di mercato e trasferirli all'interno dell'impresa (Di Stefano et al., 2012, p. 1292).

17 È stato così posto in evidenza l'importante ruolo, che viene assolto dalle relazioni noncompetitive tra le imprese e fra le imprese ed altri soggetti dell'ambiente, nel processo di generazione-diffusione delle innovazioni tecnologiche. Vari studi teorici ed empirici (degli anni ottanta e novanta) hanno dimostrato che l'innovazione tecnologica tende sempre meno ad essere il risultato di attività, condotte esclusivamente all'interno dei laboratori di R\&S di una singola impresa. Nell'attuale economia della conoscenza, l'innovazione è sempre più il risultato di attività svolte dalle imprese industriali in stretta cooperazione ed interazione con risorse complementari di altri soggetti esterni (Università, centri di ricerca scientifica-tecnologica, altre imprese, utenti). Lo sviluppo di rapporti cooperativi con altri soggetti, operanti anche in altri Paesi, è favorito da vari fattori, di cui ne evidenziamo almeno due:

a) i «limiti all'internalizzazione» delle risorse, necessarie a governare l'evoluzione tecnologica nella singola impresa;

b) la diffusione di strategie competitive, le quali dovendo affrontare processi di "concorrenza globale", sono anche orientate a ricercare alleanze con aziende straniere e accordi con strutture economiche estere. 
dell'innovazione (Chesbrough, 2003; Gassmann, 2006). Essa consiste nell' utilizzo di "flussi di conoscenza" in entrata ed in uscita, rispettivamente, per accelerare i processi di innovazione e per espandere i mercati tramite un utilizzo esterno dell'innovazione (Chesbrough e Chrowther, et al., 2006, p. 1).

Tradizionalmente le grandi imprese hanno fondato lo sviluppo di nuovi prodotti su attività di Ricerca e Sviluppo svolte internamente. In molti settori industriali, ampi laboratori di "ricerca e sviluppo" rappresentavano un investimento strategico e costituivano un importate barriera all'entrata per potenziali rivali. Di conseguenza, le grandi imprese con ampie capacità di ricerca e sviluppo e risorse complementari potevano conseguire migliori performance, rispetto ai rivali di dimensione minore (Teece, 1986). Questo processo, nel quale le grandi imprese scoprono, sviluppano e commercializzano le tecnologie internamente è stato definito modello "chiuso" di innovazione (Chesbrough, 2003).

Sebbene tale modello abbia dato buoni risultati negli anni passati, l'attuale contesto innovativo è cambiato; si pensi alla mobilità dei lavoratori, all'abbondanza di venture capital, alla dispersione della conoscenza fra una pluralità di organizzazioni pubbliche e private, alla riduzione del ciclo di vita dei prodotti, ecc..

Attualmente le imprese non sono più in grado di affrontare singolarmente l'innovazione, ma devono adottare approcci di innovazione alternativi (Van de Vrande et al., 2009, p. 424).

Uno schema che illustra questo concetto è presentato nella tabella 3.

In effetti, un numero crescente di PMI sono passate ad un modello di innovazione aperta, nel quale esse utilizzano percorsi alternativi esterni, per sfruttare le tecnologie e nello stesso tempo per acquisire la conoscenza da fonti esterne (Chesbrough, 2003).

L'innovazione aperta ha ricevuto una crescente attenzione nella letteratura scientifica dagli anni duemila. La maggior parte degli studi si sono tuttavia concentrati su imprese di grande dimensione operanti in settori high tech (Dodgson et al., 2006; Di Minin et al., 2010).

Per comprendere meglio in quale modo si articoli concretamente il processo di apertura, Dahlander e Gann (2010) propongono di utilizzare le dimensioni dell'innovazione in-bound versus out-bound e le interazioni pecuniarie versus quelle non pecuniarie ${ }^{18}$.

Pochi studi hanno cercato di esaminare empiricamente la diffusione di pratiche di innovazione aperta nelle PMI ${ }^{19}$.

18 Lichtenthaler e Lichtenthaler (2009) individuano invece tre processi cognitivi (esplorazione, ritenzione e sfruttamento della conoscenza) che possono essere realizzati internamente o esternamente. Un'ulteriore suddivisione delle pratiche di open innovation considera infine il diverso livello di apertura dei processi di innovazione e dei risultati di tali processi (Huizingh, 2010, p. 2).

19 Comunque, in relazione alla diffusione delle pratiche di open innovation nelle PMI è stato verificato recentemente da Van de Vrande et al (2009) in un interessante studio il livello di diffusione delle pratiche di open innovation in un campione di 605 imprese olandesi di due classi dimensionali (10-99 addetti e 100-499 addetti). L'analisi ha posto in evidenza 
Tab. 3: Principali processi di innovazione "aperta"

In base ad estese analisi empiriche sulle imprese di diversa classe dimensionale Gassman e Enkel (2004), sostengono, ad esempio, che l'innovazione aperta può comprendere tre principali tipi di processi.

a) Il processo "outside-in", mediante il quale l'impresa cerca di incoraggiare e sfruttare i flussi di conoscenza provenienti dall'esterno al fine di integrarli nelle attività interne di innovazione: ciò deriva dalla convinzione che il luogo, dove avviene l'innovazione, può essere diverso dal luogo nel quale viene generata la conoscenza.

b) Il processo "inside-out", attraverso il quale l'impresa utilizza canali esterni, per trasferire le proprie idee al mercato: ciò riflette la convinzione che il luogo di sfruttamento di una idea può essere diverso da quello nel quale essa viene generata.

Il processo “coupled”, nel quale l'impresa combina i due processi precedentemente considerati, mediante una collaborazione stretta con altri innovatori: quest'ultimo processo è caratterizzato da una profonda cooperazione con gli stessi partner per un lungo periodo di tempo.

Questi processi possono inoltre avere diversi livelli di apertura verso l'esterno.

Fig. 4: Principali processi di innovazione aperta

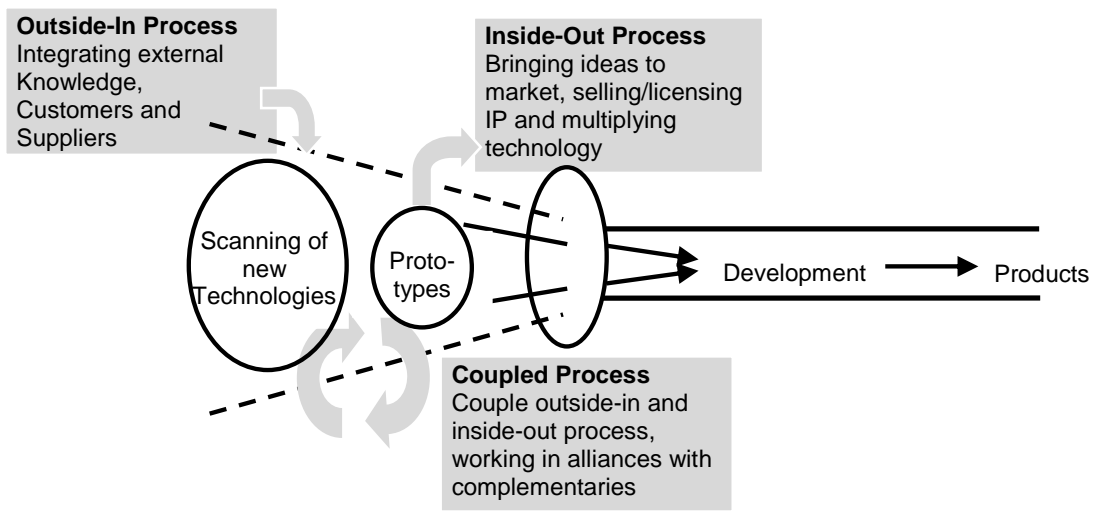

Fonte: Gassman e Enkel, 2004.

Il motivo principale che spinge la maggior parte delle imprese ad abbracciare l'open innovation è "market-related". L'impiego di queste prassi viene considerato un modo per seguire più da vicino gli sviluppi del mercato e per andare incontro alla domanda dei clienti (Baccarani, 2005). Ciò può infatti tradursi in una crescita del

che l'open innovation è rilevante, non solo nelle imprese multinazionali, ma anche nelle $P M I$. Le prassi più diffuse sono risultate quelle relative al coinvolgimento dei clienti (97\%), alle reti di relazioni esterne (94\% delle imprese) e al coinvolgimento dei dipendenti (93\% delle imprese). Dai dati emerge tuttavia una differenza significativa nell'adozione di queste prassi gestionali fra imprese di diversa dimensione. Le imprese medie adottano in maggior misura prassi di "open innovation", rispetto a quelle più piccole. Le prime dispongono in realtà di adeguate risorse e capacità organizzative per svolgere una più ampia varietà di attività innovative, rispetto alle prime, e di conoscenze interne, che possono essere utilizzate in attività di open innovation (technology exploitation). 
fatturato, in migliori risultati finanziari o in un incremento della quota di mercato (Van de Vrande et al., 2009, p. 432).

Dal punto di vista dell'analisi economica, il concetto di open innovation è relativamente nuovo, ampio e non ben definito. Dopo aver analizzato 150 paper sull'innovazione aperta Dahlander e Gann (2010), rilevano che i ricercatori tendono ad utilizzare definizioni diverse e pongono l'attenzione su aspetti diversi. Ciò rende ovviamente difficile sviluppare un corpo di conoscenze coerenti e impedisce l'elaborazione di adeguate teorie: occorre pertanto sviluppare appropriati schemi interpretativi.

\section{Partecipazione degli "innovatori volontari" ai processi di innovazione e "crowdsourcing"}

Riguardo alle problematiche attinenti alle fonti dell'innovazione, interessanti appaiono alcuni studi recenti, che hanno analizzato le caratteristiche e le motivazioni, che inducono gli "innovatori volontari" a partecipare a progetti di innovazione proposti dalle imprese (Raasch e Von Hippler, 2013).

Le ricerche hanno mostrato che gli individui sono spinti a partecipare volontariamente ad un progetto di innovazione da tre principali benefici ${ }^{20}$. Due di questi, cioè quello derivante dall'utilizzazione o dalla vendita dell'innovazione, sono collegati al risultato conseguito dal processo di innovazione (output related benefits). Il terzo beneficio, spesso trascurato nella letteratura convenzionale sull'innovazione, è collegato alla gratificazione che l'innovatore volontario consegue dalla diretta partecipazione al processo di innovazione (innovation process benefits). Quest'ultimo tipo di beneficio non sarebbe conseguito dagli innovatori volontari, se la soluzione innovativa venisse fornita all'innovatore da qualcun altro. Esempi importanti di "innovation process benefits" sono: il divertimento (e l'apprendimento) generato dalla partecipazione al progetto innovativo, il desiderio di aiutare gli altri o la reputazione derivante dall'aver realizzato contribuiti innovativi rilevanti (Raasch e Von Hippler, 2013, p. 34) ${ }^{21}$.

20 I primi studi volti a verificare il mix di benefici sperimentati dagli innovatori volontari hanno cercato di verificare perché programmatori particolarmente qualificati erano spinti a partecipare gratuitamente alla creazione di programmi open source di elevato valore operativo. Il beneficio indicato più frequentemente era collegato all'utilizzo dei programmi, che essi avevano sviluppato. Altre importanti motivazioni erano tuttavia riconducibili al divertimento e all'apprendimento derivante dallo sviluppo dei nuovi codici (Von Krogh et al., 2012).

21 Poiché gli innovatori volontari sono disposti a fornire un contribuito significativo ad un progetto motivati in parte o esclusivamente dai benefici derivanti dalla partecipazione diretta al processo di innovazione, lo sponsor del progetto può dedicare al progetto una maggiore quantità di lavoro, poiché l'innovatore non viene pagato in relazione a tutto il periodo di tempo dedicato al progetto (Raassh e Von Hippler, 2013, p. 37).

Nel processo di coinvolgimento occorre comunque fare attenzione al fatto che i volontari - che ottengono una gratificazione legata esclusivamente alla partecipazione nel progetto 
In certi casi $i$ volontari sono indotti a partecipare al progetto di innovazione, anche quando non sono interessati all'utilizzo del prodotto.

Un originale metodo di coinvolgimento nel processo organizzativo dell'innovazione è il "crowdsourcing", che si inserisce nel paradigma della "open innovation". Esso costituisce una modalità, mediante la quale l'impresa può accedere all'intelligenza distribuita della "folla", e quindi aprire i propri processi a soggetti esterni, in primis ai clienti. Nel dettaglio, il crowdsourcing è stato definito come "l'atto di un'impresa o di un'istituzione di esternalizzare un'attività o una funzione, precedentemente svolta dal proprio personale, ad una rete ampia e indefinita di persone, sotto forma di una open call" (Howe, 2006). Esso è quindi una forma di outsourcing, ma non verso altre imprese, bensì verso una massa anonima di individui. Tre sono le tipologie di soggetti coinvolti:

- gli individui, che formano la "folla" e che sono chiamati a svolgere una qualche attività;

- l'impresa, che si rivolge alla "folla" per ottenere la collaborazione su una qualche attività;

- una piattaforma di intermediazione (generalmente online), che connette l'impresa alla "folla" e che funge quindi da abilitatore delle relazioni (ad esempio, Zooppa, Best Creativity).

In concreto, l'impresa si affida agli utenti della Rete per l'ideazione di un nuovo spot pubblicitario, di un nuovo logo, del nome o delle caratteristiche di un prodotto, potendo così sfruttare l'enorme potenziale creativo esistente in Rete.

Gli effetti di tale pratica sono indicati nella tabella 4.

\section{Tab. 4: Vantaggi e svantaggi del crowdsourcing}

I principali vantaggi che un'impresa può conseguire adottando questo metodo sono:

1. il risparmio nei costi, poiché l'eventuale remunerazione dei partecipanti alla "open call" è sicuramente più bassa, rispetto a quella dovuta ad un'impresa o ad un professionista per lo svolgimento della medesima attività;

2. la migliore qualità ottenibile per il fatto che ci si rivolge non ad una specifica organizzazione, ma ad un vasta rete di individui, dove è probabile che possano emergere le idee più creative;

3. la riduzione del rischio, poiché le attività non sono affidate ad una sola impresa, e il contratto con l'individuo-fornitore viene stipulato soltanto ex post, dopo aver valutato l'operato di quello;

4. la più rapida e facile diffusione dell'innovazione sul mercato: il fatto che i partecipanti siano anche $i$ potenziali acquirenti del prodotto aumenta la probabilità di accettazione del nuovo prodotto da parte del mercato e, per effetto del passaparola che può generarsi tra gli utenti, la diffusione dell'innovazione può essere significativamente più ampia.

A fronte di questi vantaggi, è evidente che ci sono anche delle problematiche da affrontare (Schenk e Guitard, 2011, p. 103): si tratta di un'operazione complessa, la cui riuscita dipende dalla capacità dell'impresa di stimolare una certa massa critica di contributi e di articolare in maniera chiara e trasparente la proposta, in modo da evitare incomprensioni da parte dei partecipanti e la sensazione di essere manipolati o di non essere sufficientemente remunerati per il loro contributo (Djelassi e Decoopman, 2013, p. 690).

(innovation process benefits) - possono porre poca attenzione alla qualità del nuovo prodotto alla cui creazione partecipano, poiché non lo hanno mai utilizzato. 
Interessante è al riguardo il caso dell'impresa austriaca "Swarovsky", produttrice di cristalli, pietre preziose e gioielli. L'azienda ha cercato di spingere i consumatori a partecipare alla progettazione di nuovi gioielli di moda e creativi con l'aiuto della Hyve Innovation Community, che è un'azienda specializzata nella creazione di siti on line di problem solving aperti a volontari. Nel 2008 la Swarovsky ha creato un sito di crowdsourcing, che offriva la possibilità ai partecipanti di ottenere benefici non monetari, quali: l'opportunità di sviluppare e mettere in vetrina i propri disegni di nuovi gioielli; commentare e votare i disegni di altri; caricare foto e avatar ed essere inclusi in un volume sulle tendenze del design degli orologi. Questa iniziativa ebbe successo: più di 1700 partecipanti caricarono più di 3000 disegni, molti di elevata qualità. Vennero assegnati alcuni premi come riconoscimento per i contributi più eccellenti, anche se ciò non era stato pianificato. Dalle indagini empiriche svolte è emerso che le persone sono spinte a partecipare a queste iniziative, perché considerano la partecipazione una esperienza particolarmente gratificante (Fuller et al., 2011).

Il coinvolgimento di innovatori volontari può pertanto contribuire a migliorare l'efficienza dei processi di innovazione aziendale.

\section{Una riflessione sulla conoscenza della gestione dell'innovazione}

In conclusione, è evidente che qualsiasi tipo di innovazione - che utilizza risorse materiali e risorse non materiali - dà origine a una nuova conoscenza che, dal punto di vista economico, costituisce una nuova risorsa intangibile (Silvestrelli, 2012). Di fatto, qualunque innovazione è collegata allo stato delle conoscenze possedute dalle singole imprese e dal sistema del valore (cioè dalla filiera).

Com'è stato correttamente osservato (Rullani, 2009, 24-27), la conoscenza viene oggi sviluppata sempre più con alleanze strategiche, che consentono ai partners di scambiare o condividere le conoscenze, in una logica di specializzazione/integrazione che - dopo la fine del fordismo - coinvolge ormai circuiti di outsourcing sempre più estesi, grazie a rapporti di mercato, alleanze strategiche, leadership di filiera e altre forme di relazione. Infatti, un po' tutta la supply chain - dai fornitori ai consumatori finali - deve essere coinvolta, se si vuole che la conoscenza sia producibile con rischi e costi ragionevoli e che possa essere valorizzata adeguatamente.

Alla fine, una conoscenza produce molto o poco valore in funzione di quello che accade in questo percorso accidentato, che si svolge per la maggior parte fuori dai confini della singola azienda.

Le strutture, di cui oggi disponiamo per sostenere la produzione e propagazione delle conoscenze impiegate nella produzione, sono infatti (per la maggior parte) strutture artificiali, che qualcuno ha dovuto progettare assumendo i costi e i rischi della loro realizzazione.

Ogni nuovo "mediatore cognitivo" - presente in questo sistema che ha richiesto innovazioni e investimenti a rischio - ha alle spalle un'evoluzione che ha premiato 
(o punito) innovatori e sperimentatori. Egli ha creato gerarchie tra first movers e followers: ha discriminato, insomma, tra i diversi attori economici e tra i diversi territori. La situazione attuale della conoscenza nasce da una storia di esperienze aziendali e di studi, e questa storia continua proiettandosi nel futuro, con le attività che verranno svolte da manager e da operatori culturali.

Non può sfuggire che spetta principalmente ai ricercatori universitari il compito impegnativo, ma affascinante, di "spostare in avanti" i confini del sapere nel campo dell'economia e gestione dell'innovazione nelle imprese.

\section{Bibliografia}

AMIT R., ZOTT C. (2001), "Value creation in e-business", Strategic Management Journal, n. 22, giugno-luglio, pp. 493-520.

ARMBRUSTER H., BIKFALVI A., KINKEL S., LAY G. (2008), “Organizational innovation: The challenge of measuring non-technical innovation in large-scale surveys". Technovation, vol. 28, n. 10, pp. 644-657.

BACCARANI C. (2005), "Le relazioni tra impresa e mercato: una questione di potere e di rispetto", Sinergie, n. 67, pp. 149-157.

BATTISTI G., STONEMAN P. (2010), "How innovative are UK firms? Evidence from the fourth UK community innovation survey on synergies between technological and organizational innovations", British Journal of Management, vol. 21, n. 1, pp. 187206.

CALIA R.C., GUERRINI F.M., MOURA G.L. (2007), "Innovation network: from technological development to business model reconfiguration", Technovation, vol. 27, n. 8 , pp. $426-432$

CAMISON C., VILLAR-LOPEZ A. (2011), "Non-technical innovation: organizational memory and learning capabilities as antecedent factors with effects on sustained competitive advantage", Industrial Marketing Management, vol. 40, n. 8, pp. 1294 1304.

CAMISON C., VILLAR-LOPEZ A. (2014), "Organizational innovation as enabler of technological innovation capabilities and firm performance", Journal of Business Research, vol. 67, n. 1, pp. 2891-2902.

CERNE M., JAKLIC M., SKERLAVAJ M. (2013), "Decupling management and technological innovation: resolve the individualism-collectivism controversy", Journal of International Management, vol. 19, n. 2, pp. 103-117

CHESBROUGH H. (2010), "Business Model Innovation: opportunities and barriers", Long Range Planning, vol. 43, n. 2-3, pp. 354-363.

CHESBROUGH H.W. (2003), Open innovation: the new imperative for creating and profiting from technology, Harvard Business School Press, Boston.

CHESBROUGH H., CROWTHER A.H. (2006), "Beyond high tech: early adopters of open innovation in other industries", $R \& D$ Management, vol. 36, n. 3, pp. 229-236.

CHESBROUGH H., ROSEMBLOOM R.S. (2002), "The role of business model in capturing value from innovation: evidence from Xerox Corporation's technology spin-off companies", Industrial and Corporate Chang, vol. 11, n. 3, pp. 529-555.

CHRISTENSEN C.M., RAYNOR M.E. (2004), Il dilemma dell'innovatore: la soluzione, Etas Libri, Milano. 
CONTRACTOR F.J., KUMAR V., KUNDU S.K., PEDERSEN T. (2010), "Reconceptualizing the firm in a world of outsourcing and offshoring: the organizational and geographical relocation of high-value company functions", Journal of Management Studies, vol. 47, n. 8, pp. 1417-1433.

DAGNINO G. B., D’ALLURA G. M., FARACI R., PISANO V. (2011), "La localizzazione dei processi innovativi ad alto contenuto tecnologico: il ruolo delle imprese àncora fra sistemi locali e network globali”, Sinergie, n. 84. pp. 179-207

DAHALANDER L. GANN D.M. (2010), "How open is innovation?", Research Policy, vol. 39, n. 6, pp. 699-709.

DAMANPOUR F., ARAVIND D. (2011), "Managerial innovation: conceptions, processes and antecendents", Management and Organization Review, vol. 8, n. 2, pp. 423-454.

DAMANPOUR F., WALKER R.M., AVELLANEDA C.N. (2009), Combinative effects of innovation types and organizational performance: A longitudinal study of service organizations, Journal of Management Studies, vol. 46, n. 4, pp. 650-675.

DI GREGORIO D., MUSTEEN M., THOMAS D.E. (2009), "Offshore outsourcing as a source of international competitiveness for SMEs", Journal of International Business Studies, n. 40, pp. 969-988.

DI MININ A., FRATTINI F., PICCALUGA A. (2010), "Fiat: Open Innovation in a Downturn (1993-2003)", California Management Review; vol. 52, n. 3, pp. 132-159.

DI STEFANO G., GAMBARDELLA A., VERONA G. (2012), "Technolgoy push and demand pull perspectives in innovation studies: current findings and future research directions", Research Policy, vol. 41, n. 8, pp. 1283-1285.

DJELASSI S., DECOOPMAN I. (2013), "Customer' participation in product development through crowdsourcing: issues and implications", Industrial Marketing Management, vol. 42, n. 5, pp. 683-692.

DODGSON M., GANN D., SALTER A. (2006), "The role of technology in the shift towards open innovation: the case of Procter \& Gamble”, $R \& D$ Management, vol. 36, n. 3, pp. 333-346.

FAGERBERG J., FOSAS M., SAPPRASERT K. (2012), "Innovation: exploring the knowledge base", Research Policy, vol. 41, n. 7, pp. 1132-1153.

FAGERBERG J., VARSPAGEN B. (2009), "Innovation studies - The emerging structure of a new scientific field", Research Policy, vol. 38, n. 2, pp. 218-233.

FULLER J., HUTTER K., FAULLANT R. (2011), "Why co-creation experience matters? Creative experience and its impact on the quantity and quality of creative contributions", R\&D Management, vol. 41, n. 3, pp. 259-273.

GAMBARDELLA A. (1999) (a cura di), L'impresa: "vecchie" e "nuove” fonti del vantaggio competitivo, Giappichelli, Torino.

GASSMANN O. (2006), "Opening up the innovation process: towards an agenda", $R \& D$ Management, vol. 36, n. 3, pp. 223-228.

GASSMANN O., ENKEL E. (2004), "Toward a theory of open innovation: three core process archetypes", Paper presented at R\&D Management Conference, Lisbon 6 luglio.

GOLINELLI G.M. (2000), L'approccio sistemico al governo dell'impresa (volume primo, L'impresa sistema vitale), Cedam, Padova.

GOVIDARAJAN V., GUPTA A.K. (2001), "Strategic innovation: a conceptual road map", Business Horizons, vol. 44, n. 4 , pp. 3-12.

GREGORI G.L. (2001), L'evoluzione del processo di outsourcing nelle strategie di sviluppo delle imprese, Giappichelli, Torino.

HAMEL G. (2006), "The why, what and how of management innovation", Harvard Business Review, February, pp. 72-84. 
HAMEL G. (2007). The future of management, Harvard Business School Press, Boston.

HAMEL G. (2009), "Management innovation”, Leadership Excellence, vol. 26, n. 5

HOWE J. (2006), "The rise of crowdsourcing", Wired Magazine, vol. 14, n. 6 pp. 1-5.

HUIZINGH E.K.R.E. (2010), "Open innovation: state of the rate and future perspectives", Technovation, vol. 31, n. 1, pp. 1-5.

KIM W.C., MAUBORGNE R. (1997), "Value innovation: the strategic logic of high growth", Harward Bsuiness Review, vol. 75, n. 1, pp. 102-112.

KIM W.C., MAUBORGNE R. (1999), "Strategy, value innovation and the knowledge economy", Sloan Management Review, vol. 40, n. 3, pp. 41-54.

KLINE S., ROSENBERG N. (1986), “An overview of innovation”, in Landu R., Rosenberg N. (a cura di), The positive sum strategy, National Academy Press, Washington D.C., 1986.

LAMPEL J., BHALLA A. (2011), "Living with offshoring: the impact of offshoring on the evolution of organizational configurations", Journal of World Business, vol. 46, n. 3 , pp. 346-358.

LICHTENTHALER U., LICHTENTHALER E. (2009), “A capability-based framework for open innovation: complementing absorptive capacity", Journal of Management Studies, vol. 46, n. 8, pp. 1315-1338.

LUMPKIN G.T., DESS G.G. (1996), "Clarifying the entrepreneurial orientation construct and linking it to performance", Academy of Management Review, vol. 21, n. 1, pp. 135 172.

MARTÍNEZ-ROS E., LABEAGA J.M. (2009). "Product and process innovation: Persistence and complementarities", European Management Review, vol. 6, n. 1, pp. 64-75.

MATTHYSSENS P., VANDENBEMPT K., BERGHMAN L. (2006), "Value innovation in business markets: breaking the industry recipe", Industrial Marketing Management, vol. 35, n. 6, pp. 751-761.

NANUT V., TRACOGNA A. (2003), "Processi di internazionalizzazione delle imprese: vecchi e nuovi paradigmi", Sinergie, n. 60, pp. 11-34.

NELSON R.R., WINTER S.G. (1982), An Evolutionary theory of economic change, Harvard University Press, Harvard.

PAVITT K. (1991), "Alcuni fondamenti di una teoria della grande impresa innovativa", in Giannetti R., Toninelli P. (a cura di), Innovazione, impresa e sviluppo economico, Il Mulino, Bologna.

PIVA M., SANTARELLI E., VIVARELLI M. (2005), "The skill bias effect of technological and organizational change: evidence and policy implications", Research Policy, vol. 34, n. 2, pp. 141-157.

PORTER M.E. (1987), Il vantaggio competitivo, Edizioni di Comunità, Milano (trad. it. di Competitive Advantage, The Free Press, New Yok, 1985).

RAASCH C., VON HIPPLER E. (2013), "Innovation process benefits: the journey as reward", MITSloan Management Review, vol. 55, n. 1, pp. 33-39.

ROSENBUSCH N., BRINCKMANN J., BAUSCH A. (2011), "Is innovation always beneficial? A meta-analysis of the relationship between innovation and performance in SMEs", Journal of Business Venturing, vol. 26, n. 4, pp. 441-457.

RULLANI E. (2009), "Rischio e valore della conoscenza - la nuova impresa della produzione immateriale", relazione presentata al $32^{\circ}$ Convegno dell'AIDEA, Università Politecnica delle Marche, Ancona, 24-25 settembre.

SAWHNEY M., WOLCOTT R.C., ARRONIZ I. (2006), "The 12 different ways for companies to innovate, MITSloan Management Review, vol. 47, n. 3, pp. 75-81. 
SCHENK E., GUITTARD C. (2011), "Towards a characterization of crowdsourcing practices", Journal of Innovation Economics \& Management, vol. 1, n. 7, pp. 93-107.

SCHLEGELMICH B., DIAMANTOPOULOS A., KREUZ P. (2003), "Strategic innovation: the construct, its drivers and its strategic outcomes", Journal of Strategic Management, vol. 11, n. 2, pp. 117-132.

SCHMEISSER B. (2013), "A systematic review of literature on offshoring of value chain activities, Journal of International Management, vol. 19, n. 14, pp. 390-406.

SILVESTRELLI S. (1978), "Modello di sviluppo industriale per disintegrazione verticale del settore: 1'industria dei mobili in Italia", Economia e politica industriale, vol. 5, n. 18, pp. 109-143.

SILVESTRELLI S. (2004), "L'innovazione nei settori industriali: implicazioni di mercato e nuove logiche concorrenziali", Sinergie, 64-65, pp. 25-64.

SILVESTRELLI S. (2012), Decisioni strategiche di impresa, Esculapio, Bologna

TEECE D.J. (1986), "Profiting from technological innovation: Implications for integration, collaboration, licensing and public policy", Research Policy, vol. 15, n. 6, pp. 285305.

TEECE D.J. (2009), "Business Models, Business Strategy and Innovation”, Long Range Planning, vol. 43, n. 2-3, pp. 172-194.

VAN DE VRANDE V., DE JONG J.P.G., VANHAVERBEKE W., DE ROCHEMONT M (2009), "Open innovation in SMEs: trends, motives and management challenges", Technovation, vol. 29, n. 6-7, pp. 423-437.

VARALDO R. (1979), (a cura di), Ristrutturazioni industriali e rapporti tra imprese, Franco Angeli, Milano.

VARALDO R. (2006), "Il nuovo modello competitivo e aziendale dei distretti industriali", Economia e politica industriale, vol. XXXIII n. 1, pp. 25-42.

VON HIPPEL E. (1978), "Successful industrial products from custom ideas", Journal of Marketing, gennaio vol. 42, n. 1, pp. 39-49.

VON HIPPEL E. (1987), "Cooperating between rivals: informal know how trading", Research Policy, vol. 16, n. 6, pp. 291-302.

VON HIPPEL E. (1990), Le fonti dell'innovazione, McGraw Hill, Milano.

VON KROGH G., HAFLIGER S., SPATH S., WALLIN M. (2012), "Carrots and rainbows: motivation and social practice on open source software development", MIS Quarterly, vol. 36, n. 2, pp. 649-676. 